\title{
Investigation of Dependence of Power Transfer on Frequency in the Linac4 Negative Hydrogen Ion Source*)
}

\author{
Wakaba KOBAYASHI, Kenjiro NISHIDA, Daniel NOLL ${ }^{1)}$, Jacques LETTRY ${ }^{1)}$, Kazuo HOSHINO \\ and Akiyoshi HATAYAMA \\ Graduate School of Science and Technology, Keio University, 3-14-1 Hiyoshi, Kouhoku-ku, Yokohama 223-8522, Japan \\ ${ }^{1)}$ CERN, 1211 Geneva 23, Switzerland
}

(Received 9 January 2019 / Accepted 10 April 2019)

\begin{abstract}
Linac4 is radio frequency (RF) driven type negative hydrogen $\mathrm{H}^{-}$ion source. In the $\mathrm{RF}$ system, it is required to match the impedance between the RF-system and plasma loading. In order to match the impedance, it is essential to analyze the plasma impedance. In this study, we analyze the dependence of power transfer on the input parameters (frequency, input power and $\mathrm{H}_{2}$ gas pressure) by using the plasma impedance prediction model. The results indicate that in the case of frequency $=2 \mathrm{MHz}$, which is often used in the experiment, efficient power supply is seen in the wide range of input power and $\mathrm{H}_{2}$ gas pressure. Also, if the optimum value of input power and $\mathrm{H}_{2}$ gas pressure is input, it may be possible to reduce the influence of the frequency value on the power reflection efficiency.
\end{abstract}

(C) 2019 The Japan Society of Plasma Science and Nuclear Fusion Research

Keywords: negative ion source, RF plasma, plasma impedance, impedance mathing, equivalent circuit model, parameter survey

DOI: $10.1585 /$ pfr. 14.3401094

\section{Introduction}

CERN's new particle accelerator Linac4 [1] is a part of the upgrade of the Large Hadron Collider (LHC) accelerator chain. The requirement of Linac $4 \mathrm{H}^{-}$source is deliver $40-50 \mathrm{~mA}, 45 \mathrm{keV} \mathrm{H}^{-}$beam in the RFQ accelerator.

The power transfer efficiency between the RF generator and the ion source plasma is one of the important parameters that determine the extracted $\mathrm{H}^{-}$beam current. In order to achieve efficient power transfer, it is required to match the impedance between the RF-system and plasma loading. However, it is difficult because the impedance of the load depends on the time evolution of plasma parameters. In the previous study [2], the equivalent circuit model of RF-system and plasma loading which is so-called plasma impedance prediction model has been developed. The equivalent circuit conversion is shown in Fig. 1. This model is useful to cope with the load impedance change due to plasma formation.

In the experiment, input parameters (frequency, input power and $\mathrm{H}_{2}$ gas pressure) is decided empirically. The relationship between input parameters and power transfer efficiency is not clear.

In this study, we analyze the dependence of power transfer on the input parameters (frequency, input power and $\mathrm{H}_{2}$ gas pressure) by using the plasma impedance pre-

author'se-mail: kobayashi@ppl.appi.keio.ac.jp

*) This article is based on the presentation at the 27th International Toki Conference (ITC27) \& the 13th Asia Pacific Plasma Theory Conference (APPTC2018). diction model. This work allows us to understand the dependence of power transfer on the three input parameters and the mutual relationship among the three input parameters.

\section{Simulation Model}

The model consists of mainly two parts: 1) the equivalent circuit model of the RF plasma and RF coil, and 2) the global particle and energy balance of plasma [3]. These models are the same as those in Ref. [2] and are briefly summarized as follows.

\subsection{Equivalent circuit model}

The source chamber that contains plasma is surrounded by the coil (see Fig. 1(a)). The geometric features of the system are as follows: the radius of chamber $r_{0}=24 \mathrm{~mm}$, the length of the region covered by the RF coil $l=28.5 \mathrm{~mm}$, the number of turns of the coil $N$ $=5.5$. The coil current $I_{\mathrm{c}}$ induces the plasma current $I_{\mathrm{p}}$, which is mainly composed of electrons [3]. This inductively coupled plasma (ICP) can be described as $R L$ series circuit coupled inductively with the primary circuit (see Fig. 1 (b)). The symbol $R_{\mathrm{p}}$ denotes the resistance of plasma. The symbols $L_{\mathrm{p}}$ and $L_{\mathrm{mp}}$ are the inductance due to electron inertia and plasma inductance due to magnetic flux. The magnetic flux is created through the circular shaped plasma current.

The matching circuit (Fig. 1 (c)) is an equivalent to the circuit shown in Fig. 1 (b). The symbols $R_{\text {plasma }}$ and $L_{\text {plasma }}$ 


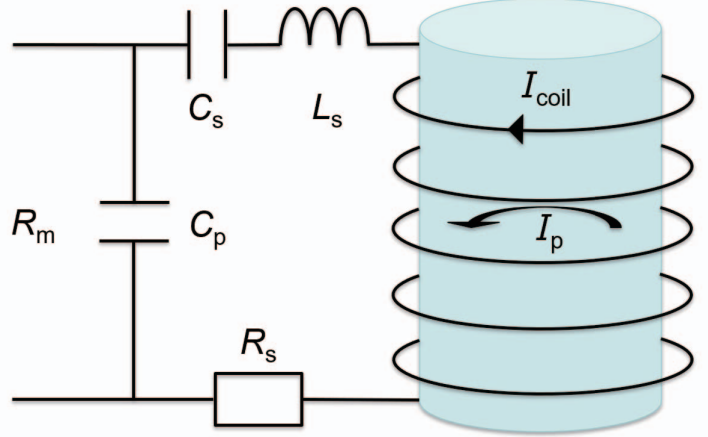

(a)

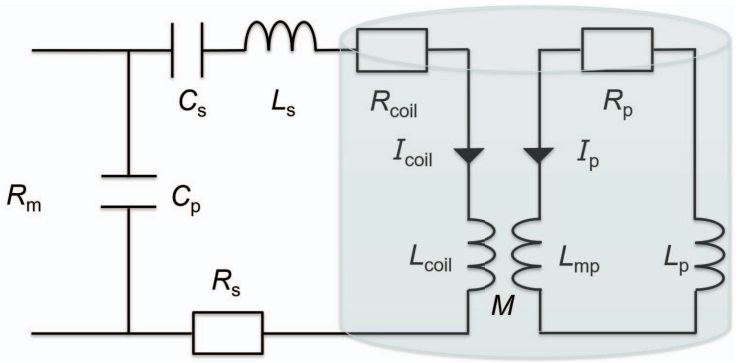

(b)

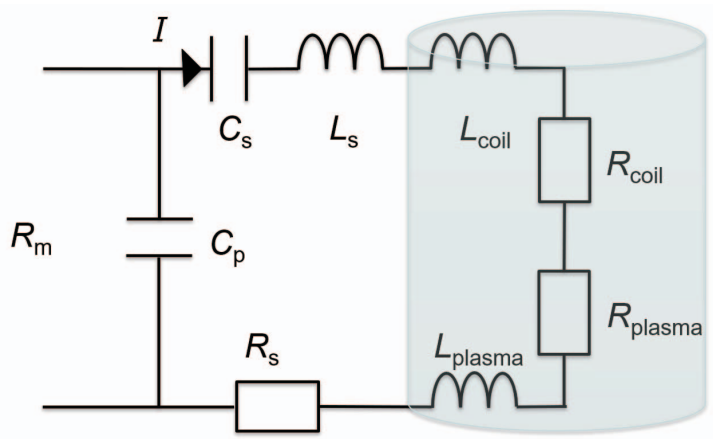

(c)

Fig. 1 (a) Matching circuit model, (b) the equivalent circuit of RF plasma inside the chamber and antenna and (c) the equivalent circuit which has been transformed into its series circuit.

denote, respectively, the resistance and the inductance of plasma after the equivalent circuit conversion. The plasma resistance $R_{\mathrm{p}}$ is modeled from the basic relation with the dissipated power in the plasma $P_{\mathrm{abs}}$ and the current. In order to obtain the expression of $P_{\text {abs }}$, it is necessary to know the electromagnetic field from the solutions of Maxwell's equations. Consequently, $R_{\text {plasma }}$ and $L_{\text {plasma }}$ can be derived as follows [3]:

$$
\begin{aligned}
& R_{\text {plasma }}=R_{\mathrm{p}}=\frac{2 \pi N^{2}}{l \omega \epsilon_{0}} \operatorname{Re}\left[\frac{i k r_{0} J_{1}\left(k r_{0}\right)}{\epsilon_{\mathrm{p}} J_{0}\left(k r_{0}\right)}\right], \\
& L_{\text {plasma }}=-\left(L_{\mathrm{mp}}+\frac{R_{\mathrm{p}}}{v_{\mathrm{m}}}\right) \frac{I_{\mathrm{p}}^{2}}{I_{\mathrm{c}}^{2}},
\end{aligned}
$$

where $v_{\mathrm{m}}$ is the sum of the collision frequencies of electrons with $\mathrm{H}$-atoms, $\mathrm{H}_{2}$-molecules, $\omega$ is the driving frequency $(\omega=2 \pi f), J_{\mathrm{n}}$ is the Bessel function of the $n$-th order, $k=k_{0} \sqrt{\epsilon_{\mathrm{p}}}$ is the wave number of the plasma. Also, the plasma permittivity $\epsilon_{\mathrm{p}}$ is given as follows:

$$
\epsilon_{\mathrm{p}}=1-\frac{\omega_{\mathrm{pe}^{2}}}{\omega\left(\omega-i \nu_{\mathrm{m}}\right)},
$$

where $\omega_{\text {pe }}$ is the electron plasma frequency which depends on the electron density $n_{\mathrm{e}}$ and electron temperature $T_{\mathrm{e}}$. Then the plasma permittivity $\epsilon_{\mathrm{p}}$ depends on the electron density and electron temperature. Both of $R_{\text {plasma }}$ and $L_{\text {plasma }}$ are dependent on the electron density and electron temperature.

\subsection{Global balance model}

We use the global particle and energy model:

$$
\begin{aligned}
& \frac{d \bar{n}_{\mathrm{j}}}{d t}=\sum_{\mathrm{k}} \bar{n}_{\mathrm{e}} \bar{n}_{\mathrm{k}} K_{\text {gain,k }}-\sum_{1} \bar{n}_{\mathrm{e}} \bar{n}_{\mathrm{l}} K_{\text {loss }, 1}-\frac{\bar{n}_{\mathrm{j}}}{\tau_{\mathrm{j}}}, \\
& \frac{d}{d t}\left(\frac{3}{2} \bar{n}_{\mathrm{e}} k_{\mathrm{B}} T_{\mathrm{e}}\right)=P_{\text {abs }}-P_{\text {loss }},
\end{aligned}
$$

where $n$ denotes density. The script $\mathrm{j}, \mathrm{k}$ and 1 represent the type of particles (electron, $\mathrm{H}, \mathrm{H}^{+}, \mathrm{H}_{2}^{+}, \mathrm{H}_{3}^{+}$). The forward difference is used. The confinement time of the $\mathrm{j}$-th particle is simply given by $\tau_{\mathrm{j}}=\frac{r_{0}}{\overline{\bar{v}_{\mathrm{j}}}}$. The symbols $K_{\text {gain }}$ and $K_{\text {loss }}$ are the rate coefficients of the particle production and the particle loss, respectively. The rate coefficients depend on the electron temperature $T_{\mathrm{e}}$. The symbol $k_{\mathrm{B}}$ is the Boltzmann constant. Whenever there is an imbalance between the power absorbed $P_{\text {abs }}$ and the power lost $P_{\text {loss }}$, there will be change in the mean energy of the electron population. The power absorbed $P_{\text {abs }}$ and the power lost $P_{\text {loss }}$ can be derived as follows:

$$
\begin{aligned}
& P_{\mathrm{abs}}=\frac{R_{\mathrm{plasma}} I^{2}}{2}, \\
& P_{\text {loss }}=\Sigma \overline{n_{\mathrm{e}}} \overline{n_{\mathrm{k}}} K E+\left(2 k_{\mathrm{B}} T_{\mathrm{e}}+q \Delta \phi\right) \frac{\overline{n_{\mathrm{e}}}}{\tau_{\mathrm{e}}},
\end{aligned}
$$

where $K$ denotes the rate coefficient and $\mathrm{E}$ denotes energy lost in single collision and $q$ denotes elementary charge. The symbol $\Delta \phi$ is the potential fall close to the wall. There are two ways for the electrons to dissipate the energy. The first term on the right side of Eq. 7 shows energy lost by undergoing collisions, transferring energy inelastically into ionization and excitation, and elastically into the thermal energy of the gas. The second term on the right side of Eq. 7 shows energy lost shows energy lost carried to the boundaries, and lost in the boundary electrostatic field or at the wall.

The current $I$ flowing the circuit and the power reflection coefficient $\Gamma$ of the matching circuit can be derived as follows:

$$
\begin{aligned}
& I=\sqrt{\frac{2 P_{\mathrm{in}}(1-\Gamma)}{R_{\mathrm{tot}}},} \\
& \Gamma=\left(\frac{Z-R_{\mathrm{m}}}{Z+R_{\mathrm{m}}}\right)^{2},
\end{aligned}
$$


Table 1 Calculation condition.

\begin{tabular}{c|c||c|c}
\hline \hline Parameters & Value & Parameters & Value \\
\hline \hline $\mathrm{H}$ temperature & $1.0 \mathrm{eV}$ & $\mathrm{H}^{+}$temperature & $1.5 \mathrm{eV}$ \\
\hline $\mathrm{H}_{2}$ temperature & $300 \mathrm{~K}$ & $\mathrm{H}_{2}^{+}$temperature & $1.0 \mathrm{eV}$ \\
\hline $\mathrm{H}_{3}^{+}$temperature & $1.0 \mathrm{eV}$ & Time step & $10^{-11} \mathrm{~s}$ \\
\hline$R_{\mathrm{S}}$ & $0.5 \Omega$ & $L_{\mathrm{s}}$ & $4.1 \mu \mathrm{H}$ \\
\hline$R_{\text {ant }}$ & $0.6 \Omega$ & $L_{\text {ant }}$ & $1.7 \mu \mathrm{H}$ \\
\hline$C_{\mathrm{s}}$ & $1.46 \mathrm{nF}$ & $C_{\mathrm{p}}$ & $5.1 \mathrm{nF}$ \\
\hline$R_{\mathrm{m}}$ & $50 \Omega$ & & \\
\hline
\end{tabular}

Table 2 The typical value of input parameters in experiments.

\begin{tabular}{c|c}
\hline Parameters & Value \\
\hline \hline $\mathrm{H}_{2}$ gas pressure & $3 \mathrm{~Pa}$ \\
Input power & $50 \mathrm{~kW}$ \\
Frequency & $2.00 \mathrm{MHz}$ \\
\hline
\end{tabular}

where $Z$ is the loading impedance including all of circuit element in Fig. 1(c). The symbol $P_{\text {in }}$ is the input power of the RF-system.

\subsection{Calculation condition}

We have calculated $n_{\mathrm{e}}$ and $T_{\mathrm{e}}$. The initial $n_{\mathrm{e}}$ is taken to be $n_{\mathrm{e}}=1.0 \times 10^{17} \mathrm{~m}^{-3}$, for ions $n_{\mathrm{H}^{+}}, n_{\mathrm{H}_{2}^{+}}, n_{\mathrm{H}_{3}^{+}}=0$ $\mathrm{m}^{-3}$ and atom $n_{\mathrm{H}}=0 \mathrm{~m}^{-3}$. At the steady state, the results do not depend on the initial ion density. The initial $T_{\mathrm{e}}$ is taken to be $T_{\mathrm{e}}=300 \mathrm{~K}$. The initial energy distributions of charged particles are assumed to be Maxwell distribution. The other parameters are constant values, which are listed in Table 1.

\section{Results and Discussion}

\subsection{Dependence of the power reflection coefficient on each input parameters}

Being based on the simulation model described in Sec. 2, the power reflection coefficient has been calculated with the basic input parameters $\left(\mathrm{H}_{2}\right.$ gas pressure, $\mathrm{RF}$ input power, and RF frequency) in Table 2. These values of input parameters are typical values used in the experiments. Then, dependence of the power reflection coefficient on these input parameters has been studied systematically in the wide range of parameter space.

\subsubsection{Dependence of the power reflection coefficient on $\mathrm{H}_{2}$ gas pressure and frequency}

Figure 2 shows the calculation results of the power reflection coefficient as a function of $\mathrm{H}_{2}$ gas pressure for various frequency. Input power is fixed the typical value $50 \mathrm{~kW}$. There is the $\mathrm{H}_{2}$ gas pressure that minimizes power reflection coefficient for each frequency. As seen from Eq. 9, the power reflection coefficient depends only on load impedance. Figure 3 shows the calculation results of load impedance as a function of $\mathrm{H}_{2}$ gas pressure for

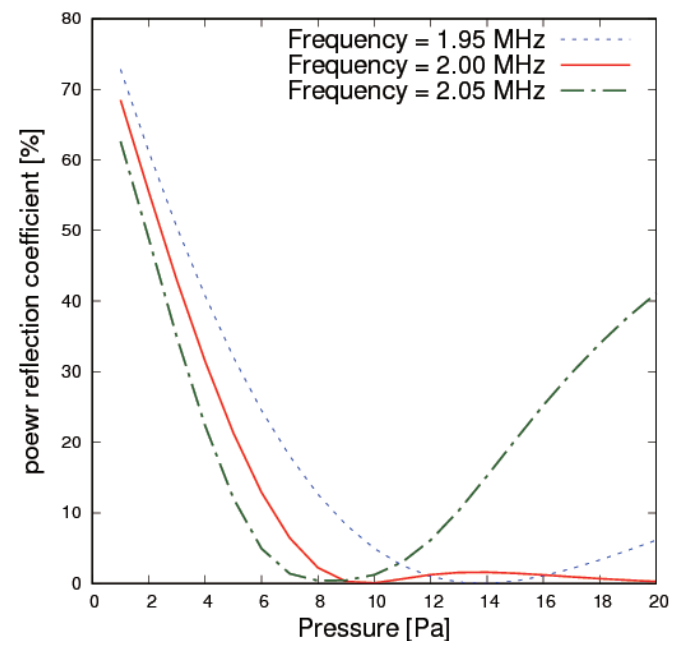

Fig. 2 The calculation results of the power reflection coefficient as a function of $\mathrm{H}_{2}$ gas pressure for various frequency.

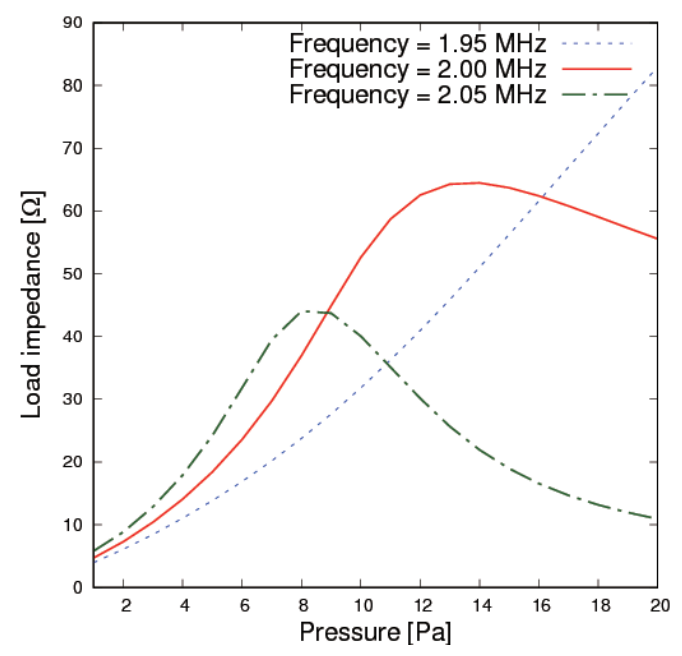

Fig. 3 The calculation results of load impedance as a function of $\mathrm{H}_{2}$ gas pressure for various frequency.

various frequency. For frequency $=2.00 \mathrm{MHz}$, the power reflection coefficient is low in a wide range because load impedance takes a value close to the impedance of coaxial cable $(50 \Omega)$. As seen from Fig. 3, the dependence of the power reflection coefficient on $\mathrm{H}_{2}$ gas pressure depends on frequency. The resonant frequency $f_{0}$ which the load impedance becomes maximum is given as follows:

$$
f_{0}=\sqrt{\frac{1}{L_{\mathrm{tot}}}\left(\frac{1}{C_{\mathrm{p}}}+\frac{1}{C_{\mathrm{s}}}\right)},
$$

where $L_{\text {tot }}=L_{\text {plasma }}+L_{\text {coil }}+L_{\mathrm{s}}$. Only plasma inductance $L_{\text {plasma }}$ is variable in Eq. 10 . Figure 4 shows the calculation results of $L_{\text {plasma }}$ as a function of $\mathrm{H}_{2}$ gas pressure for various frequency. As also shown in Ref. [4], the values of $L_{\text {plasma }}$ at the steady state do not significantly depend on the frequency. However, they depend on $\mathrm{H}_{2}$ gas pressure. When $\mathrm{H}_{2}$ gas pressure is low, the absolute values of $L_{\text {plasma }}$ 


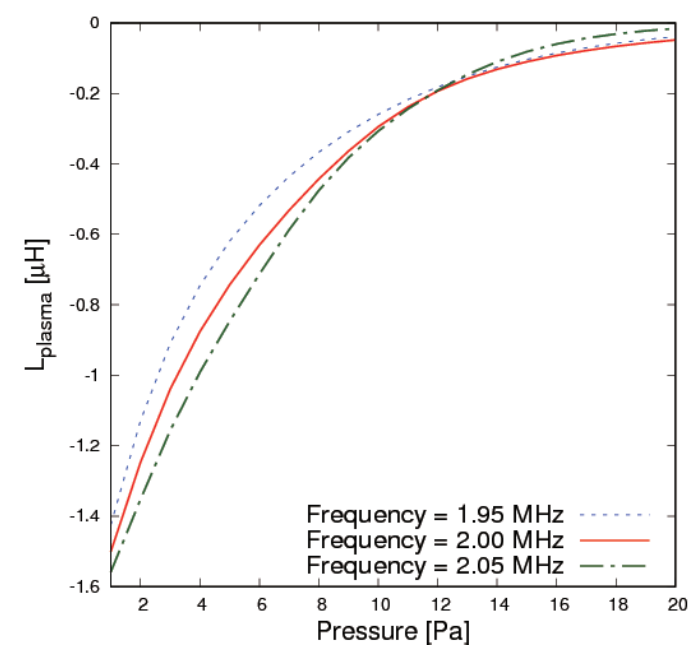

Fig. 4 The calculation results of plasma inductance as a function of $\mathrm{H}_{2}$ gas pressure for various frequency.

are close to other inductances. Since $L_{\text {plasma }}$ has a negative value, $L_{\mathrm{tot}}$ becomes low and the resonant frequency becomes high. When the $\mathrm{H}_{2}$ gas pressure is low, the resonant frequency has a value higher than the resonant frequency when there is no plasma $(1.92 \mathrm{MHz})$. For the reason, in Fig. 2, load impedance becomes higher in low pressure range when $2.00 \mathrm{MHz}$ is input. On the other hand, when $\mathrm{H}_{2}$ gas pressure is high, it takes a value less than one tenth of the other inductance. Therefore, the resonance frequency when the $\mathrm{H}_{2}$ gas pressure is high has a value close to the resonance frequency when there is no plasma (1.92 MHz). For the reason, in Fig. 2, load impedance becomes high in high pressure range when $1.95 \mathrm{MHz}$ is input.

However, it should be noted that these results have been obtained for the steady state with the relatively high initial density, i.e., they might not be correct for the low density case. It is necessary to study low density cases to make sure if it is possible to ignite the plasma or not with such a high $\mathrm{H}_{2}$ gas pressure case with $P_{\mathrm{H}_{2}}>10 \mathrm{~Pa}$.

\subsubsection{Dependence of the power reflection coefficient on $\mathrm{H}_{2}$ gas pressure and input power}

Figure 5 shows the calculation results of the power reflection coefficient as a function of $\mathrm{H}_{2}$ gas pressure for various input powers. Frequency is fixed the typical value 2.00 MHz. There is the $\mathrm{H}_{2}$ gas pressure which minimizes power reflection coefficient for each input power. When the input power is set smaller, $\mathrm{H}_{2}$ gas pressure that minimaizes power reflection coefficient is lower.

Figure 6 shows the calculation results of load impedance as a function of $\mathrm{H}_{2}$ gas pressure for various input powers. At any pressure, when $\mathrm{H}_{2}$ gas pressure is high, the load impedance becomes large. High $\mathrm{H}_{2}$ gas pressure makes electron temperature low. Since the electron temperature is low, ionization hardly occurs and electron density becomes low. In the previous study [4], the

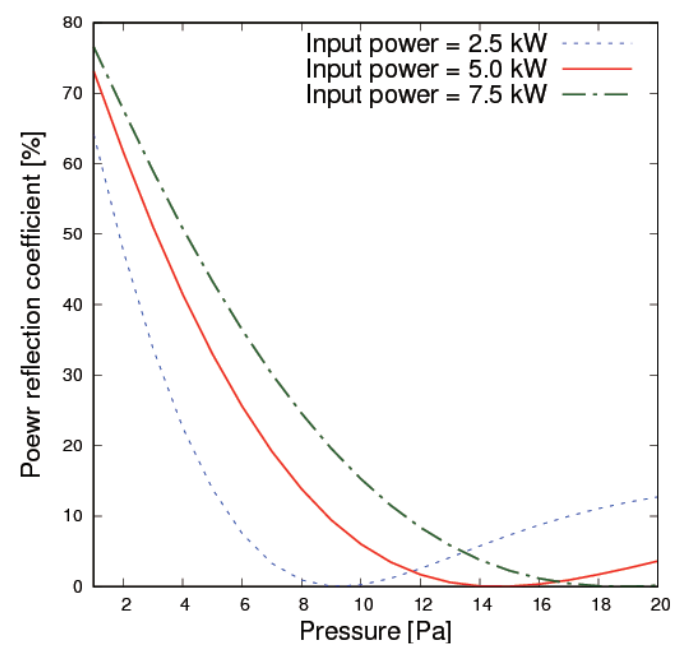

Fig. 5 The calculation results of the power reflection coefficient as a function of $\mathrm{H}_{2}$ gas pressure for various input powers.

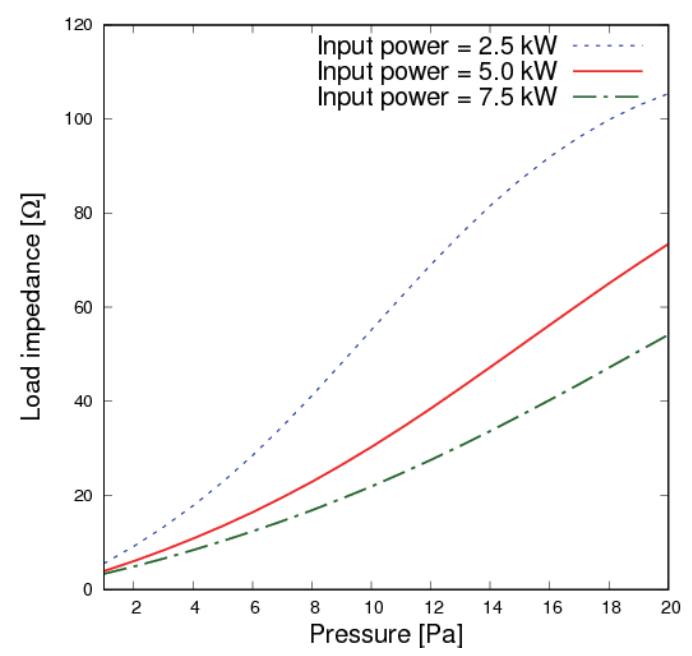

Fig. 6 The calculation results of load impedance as a function of $\mathrm{H}_{2}$ gas pressure for various input powers.

dependence of $R_{\text {plasma }}$ and $L_{\text {plasma }}$ on the electron density was clarified. When the electron density is low, $R_{\text {plasma }}$ and $L_{\text {plasma }}$ take very low values and load impedance becomes high.

Also, at any pressure, when the input power is small, the load impedance becomes large. Even in this case, electron density becomes low. Then $R_{\text {plasma }}$ and $L_{\text {plasma }}$ take very low values and load impedance becomes high.

\subsection{Dependence of the power reflection coefficient on $\mathrm{H}_{2}$ gas pressure and input power for various frequencies}

In order to clear the dependence of the power reflection coefficient on three input parameters, we have calculated the power reflection coefficient on $\mathrm{H}_{2}$ gas pressure and input power for various frequencies. The calculation results are shown in Fig. 7. 


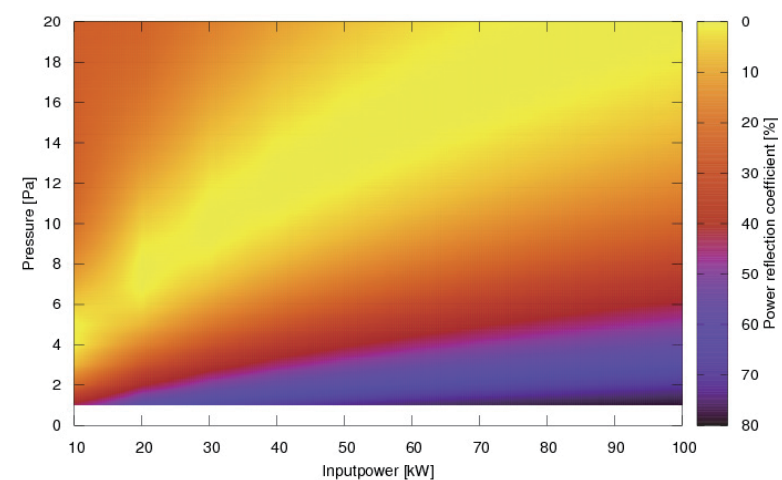

(a)

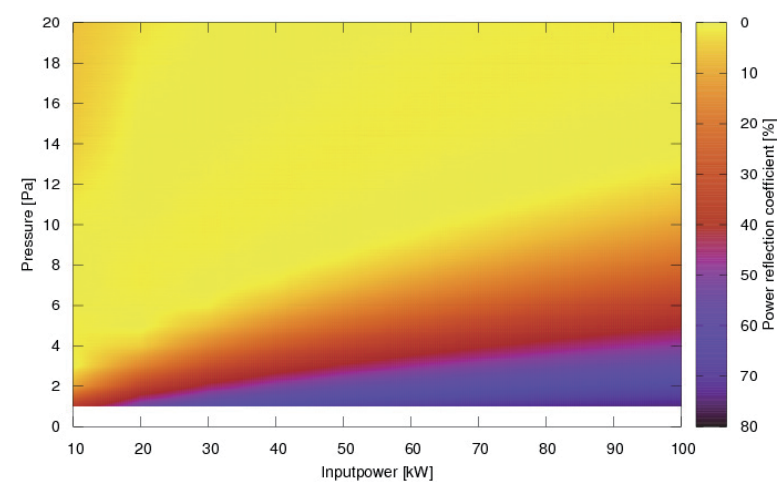

(b)

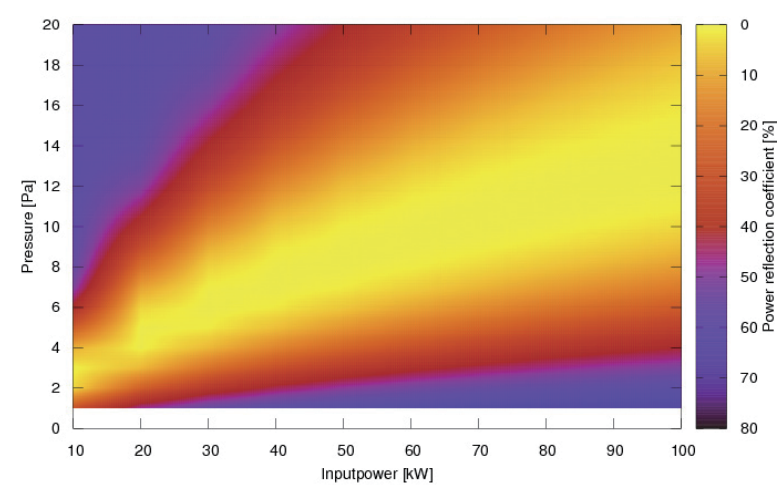

(c)

Fig. 7 The calculation results of the power reflection coefficient as a function of $\mathrm{H}_{2}$ gas pressure and input power for (a) $1.95 \mathrm{MHz}$, (b) $2.00 \mathrm{MHz}$ and (c) $2.05 \mathrm{MHz}$.

For frequency $=2.00 \mathrm{MHz}$, power reflection coefficient is low in a wide range because impedance matching is achieved in a wide range. Figure 8 shows calculation results of load impedance as a function of frequency. For input power $=20 \mathrm{~kW}$ and $\mathrm{H}_{2}$ gas pressure $=20 \mathrm{~Pa}$, quality factor which represents the sharpness of the graph representing the load impedance dependence of frequency is high and load impedance takes much larger or smaller than the impedance of coexial cable $(50 \Omega)$ depending on frequency. On the other hand, for input power $=50 \mathrm{~kW}$ and $\mathrm{H}_{2}$ gas pressure $=10 \mathrm{~Pa}$, quality factor is low and load impedance takes a value close to the impedance of coexial cable. It does not depend very much on frequency. Fig-

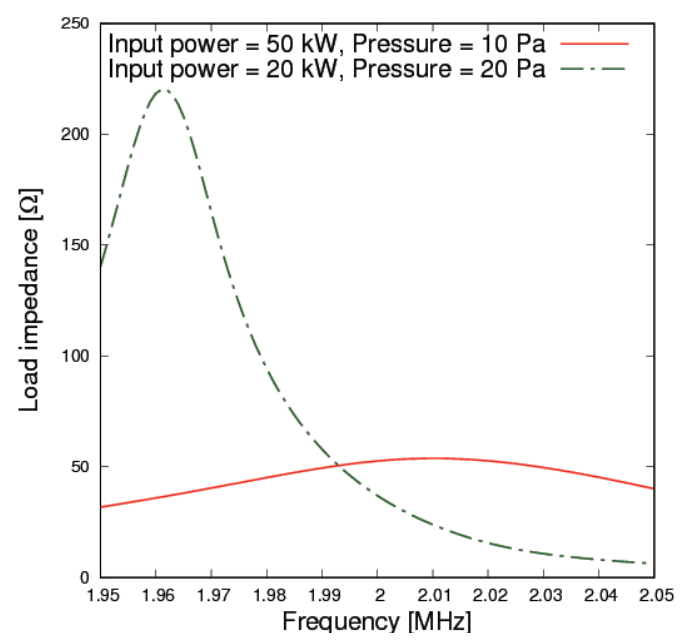

Fig. 8 The calculation results of load impedance as a function of frequency.

ure 8 shows that quality factor depends on other input parameters. If the optimum value of input power and $\mathrm{H}_{2}$ gas pressure is input, it may be possible to reduce the influence of the frequency value on the power reflection efficiency.

Also, for high $\mathrm{H}_{2}$ gas pressure and low input power range, the power reflection coefficient is high. Low input power and high $\mathrm{H}_{2}$ gas pressure make electron temperature low. For the same reason as in Section 3.1.2, impedance matching is not achieved.

Also, for the low $\mathrm{H}_{2}$ gas pressure range, the power reflection coefficient is high. In this range, the absolute value of $L_{\text {plasma }}$ is too high. Low $\mathrm{H}_{2}$ gas pressure makes low $\mathrm{H}_{2}$ density. The absolute value of $L_{\text {plasma }}$ becomes too high because low $\mathrm{H}_{2}$ density makes low collision frequency as shown in Eq. 2.

\section{Conclusion and Outlook}

In order to clear the dependence of the power reflection coefficient on each input parameters, we have calculated the power reflection coefficient using various value of each input parameters. It has been shown that the power reflection coefficient depends on value of input parameters, and input parameters are interrelated. As seen from Fig. 7, for the case of frequency $=2.00 \mathrm{MHz}$, which is often used in the experiment and particle simulation [5], power is efficiently supplied in the wide range of input power and $\mathrm{H}_{2}$ gas pressure. Also, as seen from Fig. 8, if the optimum value of input power and $\mathrm{H}_{2}$ gas pressure is input, it may be possible to reduce the influence of the frequency value on the power reflection efficiency.

Also, the plasma impedance prediction model is useful for investigating the influence of input parameters on power reflection coefficient. However, the plasma impedance prediction model may not be quantitative in some cases because of the some assumptions for simplification of the model. Morover, the model has not taken 
into account the plasma capacitance, which is important for the low plasma density regime. In order to investigate the dependence of power transfer on input parameters during plasma ignition, we are planning to take the plasma capacitance into account.

[1] J. Lettry, D. Aguglia, Y. Coutron, E. Chaudet, A. Dallocchio et al., AIP Conf. Prof. 1515, 302 (2013).

[2] W. Kobayashi, K. Nishida, S. Mattei et al., AIP Conf. Prof. 2011, 050019 (2018).

[3] P. Chabert and N. Braithwaite, Physics of Radio Frequency Plasmas (Cambridge Univesity Press, New York 2011).

[4] K. Nishida et al., Rev. Sci. Instrum. 85, $02 B 117$ (2014).

[5] S. Mattei, M. Ohta, M. Yasumoto, A. Hatayama, J. Lettry and A. Grudiev, Rev. Sci. Instrum. 85, 02B115 (2014). 